
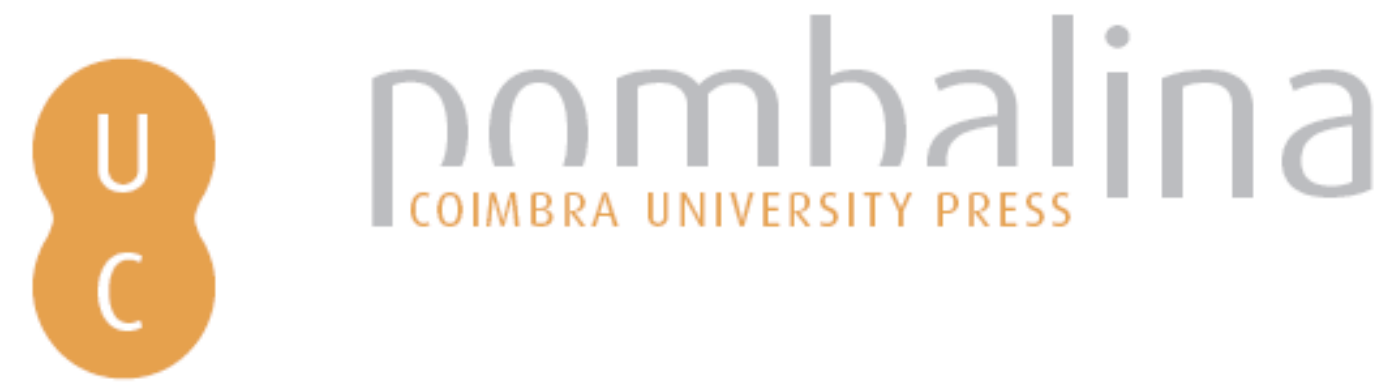

\title{
La communication politique menace-t-elle la citoyenneté? une interrogation éthique sur la communication politique nord-américaine contemporaine
}

Autor(es): $\quad$ Gauthier, Gilles

Publicado por: Imprensa da Universidade de Coimbra

URL

persistente: URI:http://hdl.handle.net/10316.2/36648

DOI: $\quad$ DOI:http://dx.doi.org/10.14195/978-989-26-0873-0_9

Accessed : $\quad$ 26-Apr-2023 10:25:21

A navegação consulta e descarregamento dos títulos inseridos nas Bibliotecas Digitais UC Digitalis, UC Pombalina e UC Impactum, pressupõem a aceitação plena e sem reservas dos Termos e Condições de Uso destas Bibliotecas Digitais, disponíveis em https://digitalis.uc.pt/pt-pt/termos.

Conforme exposto nos referidos Termos e Condições de Uso, o descarregamento de títulos de acesso restrito requer uma licença válida de autorização devendo o utilizador aceder ao(s) documento(s) a partir de um endereço de IP da instituição detentora da supramencionada licença.

Ao utilizador é apenas permitido o descarregamento para uso pessoal, pelo que o emprego do(s) título(s) descarregado(s) para outro fim, designadamente comercial, carece de autorização do respetivo autor ou editor da obra.

Na medida em que todas as obras da UC Digitalis se encontram protegidas pelo Código do Direito de Autor e Direitos Conexos e demais legislação aplicável, toda a cópia, parcial ou total, deste documento, nos casos em que é legalmente admitida, deverá conter ou fazer-se acompanhar por este aviso.

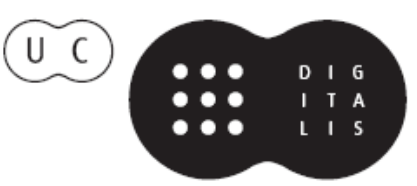


www.uc.pt/ imprensa_uc CONTACTO imprensa@uc.pt VENDAS ONLINE http://livrariadaimprensa.uc.pt JANEIRO 2015
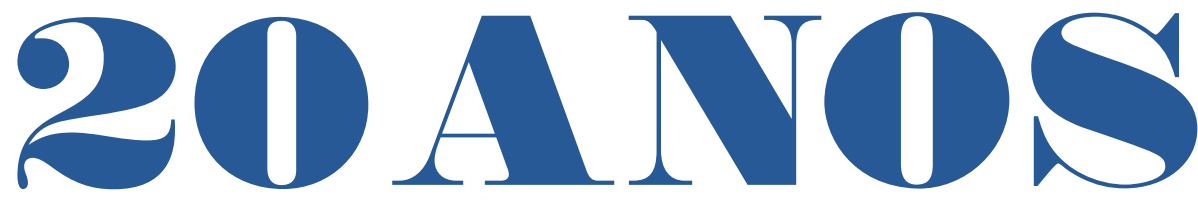

\section{DE JORNALISMO CONTRA A INDIFERENÇA}

TEXTOS DE

Marc Lits, Adriano Duarte Rodrigues, Tito Cardoso e Cunha, José Augusto Mourão, Alberto Pena Rodríguez, Maria Augusta Babo, Daniel Cronu, João Pissarra Esteves, Gilles Gauthier, Heloísa Paulo e Luís Reis Torgal, Alfredo Barroso, António Fidalgo, Nöel Nel, João de Almeida Santos, Juan Luis Cebrián, António Dias Figueiredo, Marina Themudo, Jorge Sampaio, Nelson Traquina, Mário Soares
( livro que agora se apresenta, nasce de dois desígnios fundamentais: por um lado, celebrar duas décadas de ensino do Jornalismo na Universidade de Coimbra e, por outro, partilhar com um público mais alargado um conjunto de reflexões sobre os media, o jornalismo, a comunicação e o espaço público.

Se o ensino superior do Jornalismo em Portugal, relativamente tardio em relação ao resto da Europa, deu os seus primeiros passos no fim dos anos 70 do século passado, ele aparece apenas duas décadas depois na academia coimbrã. Contudo, esta foi, no contexto nacional, a primeira licenciatura em Jornalismo, distinguindo-se, quer em título, quer em objetivos, das licenciaturas então existentes no país. A criação de uma Licenciatura em Jornalismo na Universidade de Coimbra, em 1993-1994, foi, por si, um acontecimento. Com efeito foi necessário que reitor, professores e jornalistas ousassem atualizar a oferta curricular da Faculdade de Letras, oferecendo um curso há muito desejado pela sociedade e pelo mercado, embora desconsiderado por alguns setores da academia. Correndo o risco de omitir alguém, a quem antecipadamente pedimos desculpa, não podemos deixar de recordar os esforços dos jornalistas João Mesquita, João Fonseca, em representação
Todas as gerações, sem dúvida, se julgan para refazer o mundo. A minha sabe, nc que não poderá refazê-lo. A sua tarefa é tc

Consiste em impedir que se desfaça, $p$ unicamente das suas negações A. Camus, Discursos da Suécia (1957)

do Sindicato dos Jornalistas, e de Jorge Castilho, a quem mais tarde se viria associar o nome de Mário Martins, bem como o do então Reitor da Universidade de Coimbra Rui Alarcão, e dos professores João Roque e Luís Reis Torgal. Entre 1993 e 1996, a Licenciatura em Jornalismo funcionou com um Secretariado, que teve um papel executivo e científico nos primeiros tempos do curso na FLUC. Presidido pelo Presidente do Conselho Científico Ludwig Scheidl, este 


\section{La communication politique menace-t-elle la citoyenneté? Une interrogation éthique sur la communication
politique nord-américaine contemporaine}

Gilles Gauthier

Université Naval, Québec

"[La citoyenneté] ... concerne en fait ... tout ce qui est d'intérêt commun, implique une responsabilité collective ou participative, appelle intervention ou arbitrage des pouvoirs publics.

(...) Etre politiquement citoyen ce n'est pas seulement remplir son devoir électoral, c'est participer autant que de besoin à l'organisation, au fonctionnement et à la conduite de la société à tous les niveaux et dans tous les domaines."*

Les sociétés occidentales contemporaines sont agitées par des tensions, réelles ou apparentes, entre certains de leurs idéaux démocratiques fondateurs et de nouvelles formes d'activités qui transforment radicalement l'organisation sociale. L'une de ces supposées tensions oppose l'idée de citoyenneté et la communication politique. Aux yeux de certains, la très forte incidence communicationnelle de la pratique contemporaine de la politique se concilie mal ou même est incompatible avec le principe de citoyenneté. On peut d'ailleurs considérer que la dépréciation éthique généralisée de la communication politique qui a aujourd'hui court trouve son origine, explicitement ou implicitement, dans son prétendu irrespect des droits du citoyen. Je chercherai ici à analyser, d'un point de vue moral, cette présumée disconvenance entre le principe de citoyenneté et la communication politique nord-américaine. En fait, mon propos est d'apprécier éthiquement cette dernière en regard du principe de citoyenneté. Celui-ci me sert donc d'aune ou de critère d'évaluation de la moralité de la communication politique. Je mènerai mon examen au sujet des trois principaux problèmes éthiques que

\footnotetext{
* Conseil scientifique des Francas, Réussir (reproduit dans Rencontre. Cahiers du travailleur social, no. 85 : "De la Solidarité à la Citoyenneté", 1993.
} 
pose la communication politique nord-américaine : le mensonge, l'image et la publicité négative'.

Il s'impose auparavant de préciser, un tant soit peu, ce qui est ici entendu par "principe de citoyenneté" et par "communication politique".

Pour les besoins de la présente étude, nul n'est besoin de référer à une théorie longuement développée de la citoyenneté. Il est suffisant d'en considérer simplement la conception intuitive usuelle. L'idée de citoyenneté est concomitante à la démocratie : elle stipule non seulement que les individus humains sont des êtres sociaux mais aussi qu'ils sont partie prenante à la vie de la "cité", c'est-à-dire à l'organisation politique de la vie collective. Un citoyen, en démocratie, est un membre reconnu de la société. À ce titre, lui sont dévolus des droits dont, fondamentalement, le droit de vote mais aussi des droits plus larges à participer à la vie collective de diverses façons dans différents domaines. Ces droits constituent aussi des obligations. Le statut de citoyen, s'il permet bien de jouir d'avantages, implique également quelque exigence : dans un système démocratique, les citoyens sont, ultimement, responsables de l'organisation sociale. En gros, être citoyen, c'est détenir en partage avec ses pairs le pouvoir politique essentiel.

De son côté, dans son acception la plus répandue, l'expression "communication politique" désigne la relation particulière qui lie aujourd'hui la communication et la politique. Elle dénote ce phénomène contemporain de "communicalisation" de la politique qui fait en sorte que sa pratique est devenue fortement de l'ordre de la communication. Parmi d'autres indices de cette "communicalisation", qui constituent autant de traits de la communication politique, on repère généralement l'utilisation massive de techniques communicationnelles, une approche marketing de la politique et une médiatisation très poussée de l'expression et de la livraison des messages politiques.

Aux yeux de beaucoup, la communication politique est une perversion de la politique : elle l'instrumentaliserait, la réduirait à l'image et aux apparences, y évacurait la discussion rationnelle et l'échange idéologique et, surtout, consisterait en une tentative de contrôle et de manipulation du débat politique. Si cette critique s'avère fondée, il suit que la communication politique, au moins dans certaines de ses manifestations, contrevient au principe de citoyenneté : qu'elle fait obstacle à l'exercice des droits et devoirs reconnus

${ }^{1}$ C'est à considérer l'ensemble des études d'éthique de la communication politique qu'il est possible d'identifier ces trois problèmes. Voir Gauthier (1996). 
aux citoyens. Et alors, en raison même de cette opposition, la communication politique pourrait faire l'objet d'une condamnation morale.

C'est cette problématique qui sera maintenant analysée au sujet de trois formes ou pratiques de la communication politique nord-américaine.

\section{Le mensonge}

Le mensonge est depuis toujours un problème éthique qui donne lieu à débat. Dans une perspective déontologique stricte, telle celle de Kant, il est en soi et donc toujours immoral. Dans la perspective téléologique contraire, celle par exemple des utilitaristes, certains usages ou certaines occurrences du mensonge peuvent être considérés comme moralement justifiables. Le débat perdure encore aujourd'hui au sujet de la communication politique: certaines théories, comme celle de Bok (1978), condamnent moralement toute forme de mensonge alors que d'autres, celle par exemple de Walk et Henley (1970), lui trouvent dans certains cas quelque justification. L'étude du mensonge s'est par ailleurs élargie : s'il reste une question éthique, le mensonge fait également l'objet d'analyses sociologiques dont certaines tendent à en faire voir l'utilité sociale et même la nécessité culturelle (voir, par exemple, Barnes, 1994). Certaines théories du mensonge se transforment en théories de la détection du mensonge (celle, par exemple, d'Ekman, 1992) qui fournissent un certain nombre d'instruments permettant de le déceler et qui mettent en évidence la très grande difficulté (logique et psychologique) de mentir.

Les rapports moraux entre politique et mensonge sont perçus de manière contradictoire ou à tout le moins ambigüe. D'une part, le mensonge (associé à la tromperie, au camouflage d'informations et aux promesses non tenues) constitue certes la faute morale le plus souvent reprochée au discours politique. C'est d'ailleurs en vertu de ce caractère mensonger que le discours et la communication politiques et la politique elle-même sont aujourd'hui fortement discrédités. D'autre part, le mensonge politique est, parmi les différents types de mensonges, l'un de ceux auquel on reconnaît le plus aisément une certaine légitimité ou du moins une certaine tolérance morale. Le mensonge est fréquemment vu comme faisant partie de l'art de convaincre spécifiquement politique. Il serait un instrument de l'efficace du discours politique. On va même parfois jusqu'à prétendre qu'il est nécessaire et souhaitable et que, dans certaines circonstances, il fait même l'objet d'un devoir moral. Le mensonge politique serait normal, c'est-à-dire que mentir ferait partie de la norme du discours politique. C'est ce qui explique- 
rait l'expectation assez répandue à l'égard de la politique d'y trouver moult mensonges et aussi le fait que ne se pose pas ou se pose moins au sujet du mensonge politique une exigence de consistance : non seulement admet-on que le discours politique soit truffé de mensonges, mais aussi de mensonges contradictoires.

Comme Barnes le met en évidence, la justification d'ordre moral du mensonge politique (et aussi de l'accusation de mentir qui serait aussi répandue en politique que le mensonge lui-même) est une conception de la politique comme affrontement. En concevant la politique comme une confrontation pacifique qui remplace avantageusement la guerre, on peut être amené à tolérer le mensonge : mentir devient alors un mal moindre que tuer.

L'admission que le mensonge politique puisse être légitime conduit à la distinction et pose ainsi le problème de la discrimination entre mensonges moraux et mensonges immoraux. Cette admission ne porte toujours en effet que sur certains usages seulement du mensonge. (Personne ne défend le point de vue à l'évidence intenable qu'il serait toujours moralement justifié). Ekman propose trois principaux critères susceptibles d'opérationnaliser la distinction : la motivation, la clarté du jugement politique et la cible du mensonge.

Ces trois critères sont tous en rapport avec ou peuvent être interprétés en regard du principe de citoyenneté. Le critère de la motivation stipule qu'un mensonge commis par un politicien dans son seul intérêt personnel est moralement condamnable alors qu'un mensonge ayant pour objectif la sauvegarde de l'intérêt public ou la préservation des droits des citoyens est justifiable. Suivant ce critère, les mensonges de Nixon dans sa tentative de couverture du Watergate étaient immoraux dans la mesure où ils ne visaient que son maintien à la présidence américaine, alors que ceux de Carter pour cacher les préparatifs d'opérations militaires en vue de libérer les otages américains en Iran étaient au contraire parfaitement moraux parce que motivés uniquement par la volonté de venir en aide à des citoyens.

En vertu du critère du jugement politique, un mensonge peut être justifié dans une situation où il apparaît être un moyen parmi les plus appropriés pour contribuer à une cause juste. Ce critère est relatif au degré de nécessité du mensonge. Ekman donne comme exemples de mensonges devant être estimés immoraux suivant ce critère ceux d'Oliver North dans l'affaire de l'"Irangate". Selon Ekman, les mensonges de North sont condamnables du fait qu'ils reposent sur une évaluation outrageusement simpliste d'une situation politique complexe. Dans la mesure où, en démocratie, la nécessité 
politique ne se justifie, finalement, qu'en fonction des citoyens, le critère du jugement politique est relatif, ultimement au principe de citoyenneté.

Le troisième critère proposé pour juger de la moralité d'un mensonge est sa cible, c'est-à-dire ceux à qui on ment, les "victimes" donc du mensonge. Un mensonge destiné à un ennemi militaire pourrait (au moins dans certaines circonstances) être moralement acceptable. Au contraire, mentir à son propre peuple ou à ses commettants serait (dans la plupart des cas) moralement répréhensible. Le critère de la cible a trait lui aussi, fondamentalement, au principe de citoyenneté : il pose que c'est quand et parce qu'il dupe les citoyens qu'un mensonge est immoral.

\section{L'image}

En Amérique du Nord, la publicité, et particulièrement la publicité électorale télévisée, est l'archétype de la communication politique. C'est tellement vrai que la mise en cause morale de la communication politique porte en fait, la plupart du temps, plus spécifiquement sur la publicité : quand on critique le caractère mensonger et hyperbolique de la communication politique ou quand on l'accuse de manipulation et de distorsion de l'information, c'est surtout en référence à la publicité politique. Par exemple, le reproche fait à la communication politique de contrevenir à la discussion rationnelle et ainsi de perturber le processus démocratique vise pour une part importante, explicitement ou implicitement, la publicité. Pour un, Spero (1980) considère que la publicité politique est à cet égard intrinsèquement immorale et même la plus immorale de toutes les formes de publicité.

Aussi n'est-il pas étonnant que les principaux problèmes contemporains d'éthique de communication politique aux États-Unis, au Canada et au Québec, c'est-à-dire ceux qui apparaissent à la fois les plus aigus et qui font l'objet de l'attention de recherche la plus soutenue, ont trait à la publicité, et plus spécifiquement encore une fois, à la publicité électorale. L'affirmation est, bien sûr, quelque peu arbitraire : le journalisme politique pose lui aussi de sérieux problèmes moraux. Mais on peut dire que, pour l'essentiel, les questions d'éthique qu'il soulève lui sont moins particulières que celles que pose la publicité politique. Par exemple, le reproche de "capsulisation" de l'information politique formulée à l'égard de la presse relève d'une critique morale plus large : il n'est que l'application au journalisme politique d'un blâme plus général adressé à l'ensemble du journalisme. Au contraire, les problèmes éthiques de la publicité politique et électorale présentent, pour la plupart d'entre eux, des traits distinctifs assez nets par rapport aux questions 
morales des autres formes de publicité. C'est le cas notamment des deux principaux ${ }^{2}$ de ces problèmes, ceux de l'image et de la publicité négative : il est clair qu'ils sont particuliers et propres à la publicité politique.

Un des blâmes éthiques les plus anciens, les plus constants et les plus répandus fait à la publicité politique est de porter davantage sinon exclusivement sur l'image des politiciens plutôt que sur leurs idées. Cette critique est en bonne partie développée à partir et en marge d'une distinction entre image et contenu, en anglais entre image et issues. Il est considéré que la publicité, surtout ici encore la publicité télévisée, en privilégiant l'image, contrevient au processus démocratique en court-circuitant la discussion rationnelle des questions d'intérêt public. Deux présuppositions sont à l'origine de cette mise en cause éthique de la publicité : premièrement que la politique est ou devrait être une pratique rationnelle et deuxièmement que l'image est de nature irrationnelle.

Un traitement éthique de la question de l'image, qu'il soit ou non relatif au principe de citoyenneté, nécessite la clarification de deux questions préalables : la première, conceptuelle, a trait à la définition de l'image et, plus généralement à la définition différentielle de l'image et du contenu; la seconde question, plus factuelle et empirique, est de savoir si, effectivement, la publicité comporte de façon essentielle et spécifique, c'est-à-dire plus que d'autres types de communication politique, des éléments d'image.

Quand on oppose image et contenu pour condamner la première, il est fait référence à deux séries de choses distinctes. Le terme anglais d'“issues" dénote d'abord les enjeux des débats politiques et aussi les questions et

\footnotetext{
${ }^{2}$ Outre celle de l'image et de la publicité négative, la publicité électorale soulève toute une série de questions éthiques plus ou moins importantes: d'être une voie d'accès payante (buying access) à l'électorat, d'opérer une simplification du débat politique, de faciliter la rétention d'information et de favoriser le recours à des procédés technologiques clandestins. Certaines de ces questions font l'objet de débats moraux. Par exemple, si Kaid (1991) conçoit bien que des procédés relatifs à ces questions, comme la fausse représentation dans la diffusion de messages et l'utilisation de techniques subliminales, sont moralement condamnables, elle met aussi en évidence le fait que certaines autres pratiques n'ont pas un caractère immoral aussi évident et font l'objet moins d'une analyse que d'une dénonciation pas toujours très réfléchie. Selon Kaid, par exemple, le seul fait que la publicité politique soit payante ne la rend pas immorale : si c'était le cas, ce serait également vrai pour tout autre type de publicité et, plus généralement, pour tout rapport commercial. De même, il n'apparaît pas à Kaid que la durée très courte des messages télévisés implique forcément une plus grande dilution ou simplification du discours politique que les longues tirades des discours plus traditionnels des politiciens.
} 
problèmes faisant l'objet de l'attention et de l'intérêt de la société, mais également les positions des politiciens sur ces questions et problèmes. Les issues ont donc trait, plus largement, à toute forme de contenu ou de substance politique (idées, idéologies, propositions, projets, programmes). Quant au terme d'“image", on l'utilise (en français comme en anglais) pour désigner, en plus de l'apparence physique, l'une ou l'autre de deux autres choses : soit la personnalité ou les traits de personnalité des politiciens, ce qui est aussi dénoté par le terme anglais “character"; soit l'appel à des émotions. Le contenu est réputé faire l'objet d'un mécanisme d'ordre informatif, logique, rationnel alors que l'image serait du domaine de l'irrationnel. C'est précisément en vertu de cette nature irrationnelle de l'image que la publicité politique serait éthiquement condamnable.

Une première remarque à faire est que la définition de l'image, dans son opposition à contenu, porte sur deux aspects tout à fait différents et distincts : la mise en évidence de traits de personnalité et l'appel aux émotions constituent deux choses tout à fait différentes, qui peuvent être effectuées indépendamment l'une de l'autre et qui, surtout, n'appartiennent pas à la même ontologie. Elles ne sont pas de même nature. Par conséquent, l'image n'est pas un terme à signification univoque; il y a en fait deux concepts distincts d'image. Comme nous le verrons plus loin, ces concepts donnent lieu à deux questionnements éthiques tout aussi distincts.

En fait, l'image présente des difficultés encore plus fondamentales qui en font une notion piégée. Telle qu'elle est entendue au sujet de la publicité politique, elle relève de l'initiative des politiciens : ce dont il est alors question, c'est de quelque chose que les politiciens cherchent intentionnellement à faire valoir. Or, comme le fait remarquer à juste titre Cundy (1986), il s'agit là d'un sens dérivé d'image. Une image, dans le sens premier du terme et du concept, c'est la représentation subjective qu'on se fait d'un état de choses. L'image, en politique, c'est d'abord, grosso modo, la perception d'ensemble que se fait un électorat d'un politicien. Ce n'est qu'en un sens second que l'image peut servir à désigner la tentative délibérée d'un politicien pour infléchir ou transformer la perception qu'on a de lui.

Garramone (1986) reprend et creuse cette distinction. Selon elle, la perception d'ensemble qu'a un électorat d'un politicien, ce qu'on pourrait appeler le concept global d'image, est le résultat de deux perceptions partielles : l'une portant sur les positions et idées politiques du candidat, relatives donc aux issues ou au contenu, l'autre portant sur les traits de personnalité du candidat, c'est-à-dire donc sur l'image entendue comme la tentative de mise en valeur 
de sa personne, ce qu'on pourrait considérer comme le concept limité d'image. Pour Garramone, les éléments d'image (en ce sens restreint) de la publicité contribuent tout autant que les éléments de contenu (les positions du politicien) à façonner la perception d'ensemble que s'en fait l'électorat (son image globale). Elle reconnaît ainsi que la publicité n'est pas uniquement que de l'image : qu'elle ne porte pas seulement sur les traits de personnalité d'un candidat mais qu'elle comporte aussi (ou peut comporter) des éléments de contenu. Surtout, la perspective de Garramone corrige la considération naïve suivant laquelle la publicité politique serait immorale du fait qu'elle porte sur l'image. Les éléments d'image (concept limité) n'ont pas, a priori, un rôle plus déterminant que les éléments de contenu dans la constitution de l'image (concept global) d'un politicien. Il n'y a pas d'un côté la pureté du contenu et de l'autre la corruption de l'image (concept limité) : les positions d'un candidat contribuent tout autant que ses traits de personnalité à façonner son image (concept global). Le contenu n'est donc pas totalement d'un ordre plus logique que l'image. Dit inversement, l'image n'est pas purement irrationnelle et le contenu de nature purement informative. Au contraire, dans le processus de constitution de la perception d'ensemble d'un politicien (de son image globale), l'image (concept limité) génère une connaissance tout autant que le contenu, une connaissance sur une personnalité politique. Suivant Garramone, le reproche moral fait à l'image d'être en marge de la rationalité et même de la court-circuiter n'est donc pas mérité.

Louden (1994) va dans le même sens en allant jusqu'à récuser ou, du moins, fortement atténuer la distinction entre image et contenu. Selon lui, les deux éléments participent, en interrelation, au processus cognitif de formation de l'image globale d'un politicien : la connaissance des positions d'un candidat éclaire celle de sa personnalité et la connaissance de cette dernière permet d'extrapoler sur ses idées et attitudes. Comme Garramone, Louden est de la sorte amené à rejeter le point de vue suivant lequel l'image serait immorale parce qu'irrationnelle.

Andersen (1989) pousse encore plus loin cette idée en soutenant que ce sont les éléments de personnalité des politiciens et donc l'image (concept limité) qui sont déterminants dans la prise de décision rationnelle de l'électorat. Reprenant le concept aristotélicien d'ethos, Andersen prétend que les électeurs fondent leurs choix électoraux essentiellement sur la crédibilité des politiciens. Selon lui également, les positions des politiciens sont importantes dans la mesure où précisément elles fournissent des informations sur leur personnalité. 
Au total, la distinction entre image et contenu (entre issues et image), si tant est qu'elle puisse être maintenue, ne peut en tout cas pas être fondée sur l'idée, qui se révèle être contestable sinon tout à fait fausse, que l'image aurait un caratère irrationnel à l'opposé du contenu qui serait, lui, de nature rationnelle. Dans la mesure où c'est en vertu de cette prétendue irrationalité qu'elle est décrétée immorale, l'analyse de la question de la définition de l'image conduit de la sorte au désamorçage de sa mise en cause éthique.

C'est à la même conclusion que mène l'examen de la question plus empirique de savoir si la publicité comporte plus d'éléments d'image que de contenu par comparaison avec d'autres formes de communication politique. L'une des principales idées reçues, à ce propos, est que la publicité comporte une plus grande proportion d'éléments d'image que le journalisme politique qui, au contraire, serait essentiellement constitué d'éléments relatifs au contenu. Cette vue des choses est aujourd'hui ébranlée par un certain nombre de recherches, par exemple celles menées par Joslyn (1980) et Kern (1989), qui tendent à démontrer que la publicité politique, plus particulièrement la publicité électorale télévisée, comporte une part importante d'éléments informatifs relatifs aux enjeux et aux positions des politiciens. Certaines études comparatives, Diamond et Marin (1989), Kern (1989) et Patterson et McClure (1976), concluent même que la publicité électorale contient des éléments de contenu dans une plus forte proportion que la presse qui tend à s'intéresser moins aux positions et enjeux électoraux qu'au déroulement des campagnes électorales en développant ce qu'on appelle le "horse race syndrom" : une conception de la campagne électorale comme un affrontement apparenté à une compétition sportive du type de la course de chevaux.

Quoi qu'il en soit, il semble à peu près établi que les électeurs s'approvisionnent en informations relatives au contenu davantage dans la publicité que dans la couverture journalistique (Patterson et McClure, 1976). Au total, certains prétendent ainsi que la publicité aurait une teneur et un effet cognitifs plus importants que la presse non seulement parce qu'elle livre une connaissance sur les politiciens, donc parce qu'elle comporte des éléments d'image, mais aussi parce qu'elle comporte plus d'éléments de contenu que la presse. Aussi n'est-il pas étonnant qu'on en arrive à soutenir que la publicité exerce une fonction d'agenda-setting, c'est-à-dire d'établissement de l'ordre du jour des sujets politiques d'importance. L'analyse de Roberts et McCombs (1994) révèle ainsi que, dans le cas des élections présidentielles américaines de 1992, l'agenda publicitaire a contribué autant que l'agenda des journaux à l'agenda de la télévision, c'est-à-dire que les sujets abordés dans la publicité 
ont été repris dans les nouvelles télévisées autant que les sujets traités dans la presse écrite, et que l'agenda publicitaire a contribué de façon significative à l'agenda des journaux, c'est-à-dire que ce dont a traité la presse écrite originait pour une part relativement importante de la publicité électorale.

$\mathrm{Si}$, de la sorte, la publicité peut revendiquer un statut qui ne la limite pas à l'image mais la fait aussi porter sur le contenu, on voit mal comment on pourrait lui faire globalement grief sur le plan moral, c'est-à-dire du seul fait qu'elle porte partiellement sur l'image. En tout cas, si la mise en cause morale de la publicité se justifie toujours, elle ne devrait pas y être limitée mais au contraire logiquement aussi s'étendre, eu égard à l'opposition image-conte$\mathrm{nu}$, au journalisme politique.

Dans une perspective plus proprement éthique, pour traiter adéquatement de l'image dans la publicité politique, il faudrait démontrer en quoi la mise en valeur de traits de personnalité ou le recours aux émotions peuvent être moralement douteux. Comme nous l'avons déjà fait remarquer, il s'agit là d'aspects qui ne sont pas du tout identiques et qui doivent donc être traités indifféremment l'un de l'autre. Les modalités de ces traitements distincts présentent néanmoins des similitudes. Entendue comme valorisation de la personnalité ou comme appel aux émotions, l'image dans la publicité pose une difficulté morale essentiellement si, dans le premier cas, la personne des politiciens et, dans le second cas, les sentiments sont vus comme n'étant pas pertinents à la discussion et au débat politique. Autrement dit, ce qui préside à la reconnaissance d'un problème et d'une mise en cause éthique de la publicité, sous l'un et l'autre des deux sens d'image, c'est une conception de la communication politique (et, plus généralement, de la politique elle-même). Ceux et celles qui condamnent en bloc l'image dans la publicité politique, qui la décrètent immorale du seul fait qu'elle porte sur la personnalité des politiciens ou sur des émotions sans prendre la peine de justifier plus précisément leur jugement adhèrent, explicitement ou implicitement, à une conception normative de la communication politique suivant laquelle elle serait purement une discussion rationnelle d'idées. On comprend pourquoi, pour ceux et celles-là, la contestation morale de la publicité se cristallise dans l'opposition image - contenu.

Mais la conception normative de la communication politique ne s'impose pas d'emblée. D'aucuns pourraient soutenir une conception plus "humaniste" en prétendant que parce qu'elle a trait à l'homme, aux rapports entre les hommes et à des sujets primordiaux de leur existence, la communication politique (et, encore une fois, l'ensemble de la politique) est affaire tout au- 
tant de personnes et de personnalités et de sentiments et d'émotions, d'une part, que d'idées et d'idéologies, d'autre part. Et cela non seulement dans sa pratique effective mais aussi dans sa nature même. De ce point de vue, il devient normal, légitime et éthiquement correct que la communication politique, y compris la publicité politique, puisse avoir partiellement pour objet la personnalité des politiciens et les émotions. Un certain nombre de chercheurs, dont Diamond et Bates (1992), Shyles (1986) et Devlin (1986) soulignent positivement, sous un aspect ou un autre, la fonction exercée par la publicité de mieux faire connaître les politiciens à l'électorat.

Cela ne veut pas dire que toute mise en valeur de traits de personnalité et tout recours aux émotions soit moral. Si ce n'est pas intrinsèquement, par définition ou a priori, que l'un et l'autre sont immoraux, certaines de leurs occurrences (ou série d'occurrences) peuvent être éthiquement constestables. Le problème, alors, devient de distinguer entre les emplois ou usages moraux et immoraux des traits de personnalité et des émotions en publicité politique. Et cette distinction relève de critères appropriés : il faudrait pouvoir déterminer les conditions suivants lesquelles la mise en évidence de traits de personnalité et l'appel aux émotions sont dans certains cas moraux et immoraux dans certains autres cas.

Le principe de citoyenneté avalise cette double vue des choses. En considérant, d'abord, que le citoyen n'est pas un sujet abstrait mais un être humain affectif autant que rationnel, on doit reconnaître une certaine légitimité possible à la mise en valeur de traits de personnalité et au recours aux émotions : c'est-à-dire que ces deux aspects de l'image ne peuvent pas être a priori frappés de suspicion morale. Cette reconnaissance n'exclut cependant pas que certains de leurs usages soient éthiquement condamnables. Le principe de citoyenneté peut lui-même permettre de départager, au moins dans certains cas, les occurrences morales et les occurrences immorales de la mise en valeur de traits de personnalité et de l'appel aux émotions : la question qu'il faut poser est celle de savoir si le citoyen est ou non manipulé et donc brimé dans ses droits.

\section{La publicité négative}

Le deuxième problème éthique majeur posé par la publicité politique est celui de la publicité négative. La publicité négative est une forme particulière de publicité électorale apparue ou plutôt dont l'appellation est apparue aux États-Unis au cours des années 8o. Elle est jusqu'à maintenant restée un phénomène typiquement américain qui ne s'est pas vraiment étendu au 
Canada et au Québec. Grosso modo, une publicité négative est une publicité qui, au lieu de faire valoir la personne ou les positions d'un politicien, comme le fait une publicité qu'on pourrait à l'inverse qualifier de "positive", s'en prend plutôt à un opposant de façon agressive et violente. Voici quelques exemples devenus classiques de messages de publicité négative diffusés à l'occasion d'élections présidentielles américaines : le Daisy Girl Spot : une attaque du candidat républicain Barry Goldwater montrant une bombe qui explose dans l'oeil d'une petite fille effeuillant une marguerite après un décompte de dix à un (1964); le Grizzly Spot : une attaque du candidat démocrate Walter Mondale qui montre un ours agressif illustrant les dangers d'une attitude conciliante à l'égard de l'URSS (1984); le fameux Willie Horton Spot: une attaque du candidat démocrate Michael Dukakis portant sur la hausse de la criminalité supposément consécutive à la mise sur pied de programmes de réhabilitation de criminels (1988).

La majorité des analystes et observateurs jugent que la publicité négative est éthiquement répréhensible. Ce reproche reste cependant intuitif et relève pour une bonne part de la pétition de principe: la publicité négative est considérée comme immorale... parce que négative. C'est en vertu du sens péjoratif très fort qui colle au terme "négatif», sens péjoratif qui fait en sorte que lorsqu'on qualifie quelque chose de négatif, on donne à penser que cette chose est néfaste, répréhensible et qu'elle devrait être évitée, que la publicité négative est jugée immorale. Dans la très grande majorité des cas, la condamnation morale de la publicité négative ne consiste qu'en cette réprobation.

Comme dans le cas de l'image, le traitement éthique adéquat de la publicité négative exige un détour important sur la question de sa définition. Les premiers à définir la publicité négative, Surlin et Gordon (1977), la caractérisent comme une attaque directe d'un adversaire politique. Pour certains, cette attaque peut porter tout aussi bien sur l'adversaire lui-même, ses positions (Kem, 1989), son parti (Surlin et Gordon, 1977) et, le cas échéant, son bilan gouvernemental. Pour d'autres (Pfau et Burgoon, 1989), elle se limite à la seule attaque de la personne même de l'adversaire. D'autres chercheurs précisent que pour être négative, une publicité doit être malintentionnée : avoir pour objectif de déprécier l'adversaire (Merritt, 1984), être malicieuse ou vicieuse (James et Hensel, 1991) ou encore chercher à ridiculiser l'adversaire (Taras, 1990).

Ces différentes définitions plus ou moins apparentées présentent un même problème : elles souffrent d'un défaut d'opérationnalisation. Elles ne permettent pas de repérer tous les messages de publicité négative et non plus donc de les départager de ceux qui n'en sont pas. Quelle que soit la 
définition que l'on retienne à partir des éléments ci-haut énumérés, elle ne s'applique pas à certains messages pourtant considérés comme des prototypes de publicité négative comme le Daisy Girl Spot ou le Grizzly Spot . Dans ces messages, il n'est aucunement fait mention à Barry Goldwater et à Walter Mondale; ils ne s'en prennent donc pas, explicitement du moins, à leur personne. Les deux messages ont cependant été (généralement si ce n'est unanimement) interprétés comme une attaque des positions militaristes de Goldwater, dans le premier cas, et de la politique de conciliation avec l'URSS de Mondale, dans le second. Le Daisy Girl Spot et le Grizzly Spot sont aujourd'hui considérés comme des exemples classiques de publicité négative bien qu'ils ne répondent pas à sa définition standard.

C'est pour pallier à ce problème d'opérationnalisation que Johnson-Cartee et Copeland (1991) et Gronbeck (1994) en proposent, chacun de leur côté, une typologie générale. Ils distinguent trois différents genres de publicité négative : l'attaque directe, la comparaison directe et la comparaison implicite (respectivement appelées "direct attack ads", "direct comparison ads" et "implied comparison ads" par Johnson-Cartee et Copeland et "assault ad", "comparative ad" et "implicative ad" par Gronbeck). L'attaque directe porte exclusivement sur l'adversaire qu'elle mentionne nommément en en faisant une présentation critique. La comparaison directe fait valoir la supériorité d'un politicien sur son adversaire en mettant en évidence les défauts, carences ou échecs du second et les qualités, capacités et réussites du premier. La comparaison implicite ne nomme pas expressément l'adversaire mais cherche, à la faveur d'informations contextuelles, à développer, chez les électeurs, une perception dépréciative de cet adversaire. Le contenu de l'attaque directe et de la comparaison directe s'impose de lui-même; pour être saisi, celui de la comparaison implicite exige un travail d'inférence du destinataire qui, pour l'interpréter correctement, doit disposer des informations contextuelles nécessaires à sa compréhension. Le Daisy Girl Spot et le Grizzly Spot sont de bons exemples de comparaison implicite.

Outre un problème de définition, la publicité négative présente aussi un problème de dénomination. À ce propos, il faut d'abord faire remarquer, comme nous l'avons antérieurement indiqué, que l'expression même de "publicité négative" (negative advertising) apparaît au début des années 1980 et connaît un usage étendu à partir de l'élection présidentielle américaine de $1988^{3}$. Or, ce n'est pas parce qu'on ne parlait pas auparavant de “publicité

${ }^{3}$ Qui mettait aux prises le républicain George Bush et le démocrate Michael Dukakis et 
négative" qu'elle n'existait pas. Il serait bien difficile d'identifier le premier message politique qui s'attaquait à l'adversaire mais, chose certaine, furent diffusés bien avant 1980 des messages qui correspondent tout à fait à la caractérisation qu'on allait donner à la publicité négative. Le Daisy Girl Spot est ici encore le meilleur exemple. Il fut diffusé, rappelons-le, en 1964, ne fut alors en aucun moment baptisé de "publicité négative" mais fut néanmoins considéré comme un classique du genre quand l'emploi de l'expression s'est plus tard généralisé. Bref, le phénomène de la publicité négative est beaucoup plus vieux que sa dénomination.

Les principales difficultés que pose la publicité négative sont relatives au prédicat "négative". Évidemment, d'un point de vue intuitif, il fait sens de qualifier de "négative" une publicité dont l'objectif est de déprécier, décrier, discréditer un adversaire plutôt que de mettre un politicien en valeur. Mais, comme nous l'avons plus haut précisé, le qualificatif "négatif" comporte une connotation péjorative très forte qui dépasse ce premier sens purement technique. Normalement, quand on dit d'une chose qu'elle est "négative", on veut par là indiquer ou suggérer que cette chose est mauvaise et, le cas échéant, immorale.

La très grande majorité des critiques et des condamnations morales de la publicité négative la considèrent comme telle par nature ou par définition : sans faire de démonstration probante, on la décrète immédiatement immorale parce que négative ${ }^{4}$. Les rares raisons non tautologiques quelquefois

qui fut très certainement l'une des campagnes les plus agressives de l'histoire.

${ }^{4}$ Certains travaux prennent la défense de la publicité négative et soutiennent même qu'elle est tout à fait morale. Garramone et al. (1990) et Joslyn (1986) lui trouvent ainsi pour vertu une valeur informative importante. Johnson-Cartee et Copeland (1991) considèrent, pour leur part, qu'elle a pour avantages de créer un plus grand intérêt en dramatisant les enjeux électoraux, d'accentuer l'importance du bilan des politiciens, de stimuler la discussion publique et la couverture journalistique des campagnes électorales, de permettre une meilleure évaluation du personnel politique et d'entraîner une polarisation bénéfique qui simplifierait et faciliterait le choix électoral. Cette question de l'utilité de la publicité négative n'a évidemment rien à voir avec celle de son efficacité. Certaines études (entre autres Garramone et Smith, 1984, Kaid et Boydston, 1987, Gronbeck, 1992, Pfau, Parrott et Lindguist, 1992 et Tinkham et Weaver-Lariscy, 1993) tendent à montrer qu'elle s'avère électoralement rentable (ne serait-ce que parce qu'elle permet de détourner l'attention et de mettre l'adversaire sur la défensive et aussi parce qu'il serait plus facile de créer un mouvement de vote "contre" que "pour"). La publicité négative serait plus efficace quand elle est diffusée par un intervenant soi-disant indépendant que par l'organisation d'un politicien (Garramone, 1985 et Tinkham et Weaver-Lariscy, 1993). D’autres recherches (par exemple Hill, 1989) amènent au contraire à penser que la publicité négative n'est, somme toute, pas du tout 
avancées pour appuyer l'idée de l'immoralité de la publicité négative restent tout à fait périphériques. Certains (comme Devlin, 1989 et Spiro, 1980) soutiennent qu'en vertu de sa négativité, elle est davantage porteuse que la publicité "positive" d'informations fausses, erronées, distordues ou incomplètes. D'autres, par exemple Ansolabehere et Iyengar (1995) et Ansolabehere, Iyengar, Simon et Valentino (1994), soutiennent que la publicité négative a pour conséquence néfaste d'accroître le désintérêt, la démobilisation et le cynisme des citoyens à l'égard de la politique et du processus électoral. Mais ce ne sont là que supputations qui restent encore à être vérifiées comme le démontrent Garramone et al. (1990), Martinez et Delegal (1990) et Romanow, Soderlund, et Price (1991).

Pour déterminer de façon plus convaincante si la publicité négative est ou non morale, il faudrait établir si ses deux aspects constitutifs sont ou non éthiquement justifiés : est-il légitime de traiter de l'adversaire (plutôt que de soi-même) et de blâmer et dénigrer cet adversaire plutôt que de faire valoir sa propre personne, proposer et préconier ses propres idées? Comme dans le cas du problème éthique de l'image, la réponse à cette question renvoie à une opposition entre différentes conceptions de la communication politique (et de la politique). Une première form de cette opposition est celle-là même que nous avons déjà rencontrée au sujet de la moralité de l'image dans la publicité : celle donc entre la conception normative de la communication politique comme discussion rationnelle d'idées et sa conception plus "humaniste" qui prend acte du fait qu'elle concerne les hommes dans la totalité de ce qui les constitue. La conception normative de la communication politique implique qu'il est immoral de traiter de la personne des adversaires; la conception plus "humaniste" apporte au contraire une légitimation éthique à cette référence.

Une seconde opposition pertinente au traitement éthique de la question de la publicité négative a également pour l'un de ses termes la conception normative de la communication politique. Si la communication politique

plus efficace que la publicité "positive". Il se dégage de certaines analyses (JohnsonCartee et Copeland, 1991) que le recours à la publicité négative comporte un certain nombre de risques et même d'effets pervers : un effet boomerang (Garramone, 1984, Merritt, 1984 et Jamieson, 1992), un effet dit du "syndrome de la victime" (Garramone, 1984) et un double effet négatif (Merritt, 1984). Finalement, il ressort (Garramone, 1985) que la pire réplique qu'un politicien attaqué puisse donner à une publicité négative est de ne pas répondre. Il doit à tout prix se défendre : cela peut lui permettre à la fois de diminuer l'effet néfaste sur sa candidature et d'augmenter l'effet boomerang sur celui qui l'a attaqué. 
est vue comme pure discussion rationnelle d'idées, alors il est immoral de s'attaquer à ses adversaires. Mais une conception différente, celle en vertu de laquelle la communication politique est un lieu d'affrontement ou, comme l'écrit Dominique Wolton (1989, 30), un "espace où s'échangent [des] discours contradictoires", amène à voir les choses de façon nettement plus nuancée. Si la communication politique est envisagée comme une confrontation entre des orientations, options, projets de société non seulement différents mais aussi divergents, il est alors normal, admissible et éthiquement acceptable que les tenants de ces positions fassent valoir tout autant les faiblesses des positions adverses que la valeur et le bien-fondé de leurs propres positions.

$\mathrm{Si}$, de la sorte, la publicité négative n'est pas intrinsèquement immorale, il ne suit pas que toutes ses occurrences soient morales. Certaines attaques de l'adversaire peuvent être irrespectueuses, voire même malhonnêtes et il serait difficile de leur reconnaître quelque légitimité éthique. Au total, la publicité négative est, du point de vue éthique tout à fait semblable à l'image: elle n'est pas immorale par définition mais certains de ses usages peuvent l'être. La question, alors, toujours comme dans le cas de l'image, est de trouver des critères permettant de discriminer ses occurrences éthiques et ses occurrences immorales.

À ce propos, une première série de distinctions peuvent être dégagées des recherches menées jusqu'à maintenant sur la publicité négative. Ces distinctions peuvent être établies dans la foulée de l'opposition entre image et contenu. Certains éléments des messages de publicité négative portent sur du contenu, sur les positions et idées de l'adversaire; ils constituent ce qu'on appelle en anglais des "political issue appeals". D'autres portent plutôt sur la personne même de l'adversaire : ce sont, toujours en anglais, des "personal characteristics appeals" (Johnson-Cartee et Copeland, 1991)5. Les premiers semblent, dans l'ensemble, éthiquement acceptables; il apparaît en effet légitime que des choses comme le bilan d'un politicien, sa performance politique passée, ses positions sur les enjeux de l'heure fassent l'objet de l'attention publique. Il en va de même (ou peut en aller de même) pour certains aspects relatifs à la personnalité de l'adversaire. Sa compétence administrative, son sens du leadership, sa capacité à dégager des consensus et même à la rigueur son histoire médicale peuvent et peut-être même doivent être considérés. Il

${ }^{5}$ Des études (par exemple, Roddy et Garramone, 1988) ont montré que les messages de publicité négative qui misent sur les positions de l'adversaire plutôt que sur sa personne ont plus d'impact sur l'électorat. Peut-être celui-ci est-il enclin à admettre et à juger moraux les premiers plus que les seconds? 
n'y a donc pas d'offense éthique à y faire référence. Ce n'apparaît pas être le cas (du moins normalement) pour certains autres aspects d'ordre personnel comme la croyance religieuse, l'appartenance ethnique, l'orientation sexuelle et la vie matrimoniale. Les attaques de l'adversaire sous ces différents aspects seraient donc immorales ${ }^{6}$.

$\mathrm{Si}$, curieusement, l'utilité de ces distinctions s'avère plus immédiate pour la question de la publicité négative, probablement parce qye cette dernière met en cause la personne de l'adversaire, elles peuvent aussi être appliquées, rétroactivement en quelque sorte, à la problématique de l'image dans la publicité électorale, c'est-à-dire au sujet de la personne du politicien promoteur d'un message.

À première vue, les distinctions entre les éléments des messages de publicité négative portant sur des aspects de contenu (les political issue appeals) et ceux portant sur la personne des politiciens (les personal characteristics appeals) et, parmi ces derniers, entre des aspects probants (du type de la compétence administrative) et d'autres aspects inconvenants (comme la croyance religieuse) apparaissent peu à voir avec le principe de citoyenneté. Ils semblent, en effet, n'avoir trait qu'au personnel politique: l'adversaire, dans le cas de la publicité négative; soi-même, dans le cas de l'image. Mais ces distinctions demandent à être fondées dans des critères plus généraux qui, eux, renvoient aux citoyens et même ne peuvent être compris que relativement au principe de citoyenneté. J'ai, ailleurs, ébauché quelques-uns de ces critères pour diverses formes de communication politique : la publicité électorale dans son ensemble (Gauthier, 1994), la publicité négative (Gauthier, à paraître 1) et les débats politiques télévisés (Gauthier, à paraître 2). De cette ébauche, il se dégage que trois principaux critères permettent de distinguer les occurrences morales et les occurrences immorales de publicité négative : un critère de véridicité, un critère de justification et un critère de pertinence. Le critère de véridicité stipule que le contenu d'un message doit être vrai, le critère de justification que l'objet de l'attaque de l'adversaire puisse, au moins en principe, faire l'objet de quelque démonstration (qu'elle repose sur quelque "évidence", "données" ou "preuve") et le critère de pertinence que l'attaque soit motivée (c'est-à-dire qu'il doit y avoir quelque rapport entre ce qui est dit de négatif ou de péjoratif à l'égard de l'adversaire et la rai-

\footnotetext{
${ }^{6}$ Cette vue des choses rejoint parfaitement les positions défendues par certains philosophes contemporains sur la problématique voisine mais plus large de la raison publique. Voir Bonin (à paraître), Greenawalt (1995) et Solum (1993).
} 
son même de le dire). En dernière instance, l'application de ces trois critères ne se justifie que par rapport au principe de citoyenneté : en effet, les exigences de véridicité, de justification et de pertinence trouvent leur raison d'être ultime relativement aux citoyens.

\section{Conclusion}

Le premier résultat de l'analyse qui précède est une relativisation de la moralité des trois principaux problèmes éthiques aujourd'hui posés par la communication politique nord-américaine. Il s'avère, en effet, que le mensonge, l'image et la publicité négative ne sont pas intrinsèquement immoraux. La reconnaissance d'une distinction de principe entre leurs usages possibles, plus précisément entre leurs occurrences morales et leurs occurrences immorales, conduit à la nécessité de l'établissement de critères susceptibles de permettre le repérage des unes et des autres. Ces critères peuvent être fondés dans le principe de citoyenneté. (Probablement aussi doivent-ils l'être dans une théorie le moindrement développée de la démocratie.)

Il est possible d'en conclure que la communication politique, si elle peut bel et bien comporter quelques aspects moralement répréhensibles, n'est pas par nature ou par définition (au moins pour ce qui est du mensonge, de l'image et de la publicité négative) en contradiction avec le principe de citoyenneté.

\section{Références bibliographiques}

Andersen, K. E. (1989) : "The Politics of Ethics and the Ethics of Politics", American Behavioral Scientist, 32(4), 479-492.

Ansolabehere, S.; Iyengar, S. (1995): Going Negative. How Attack Ads Shrink and Polarize the Electorate, New York: Free Press.

Ansolabehere, S.; Iyengar, S.; Simon, A.; Valentino, N. (1994): “Does Attack Advertising Demobilize the Electorate?", American Political Science Review, 88(4), 829-838.

Barnes, J.A. (1994) : A pack of lies. Towards a sociology of lying, Cambridge: Cambridge University Press.

Bok, S. (1978): Lying: Moral Choice in Public and Private Life, New York: Pantheon. Bonin, Pierre-Yves (à paraître) : "Introduction à l'idée de raison publique" (projet d'article soumis à Communication).

Cundy, D.T. (1986) : «Political Commercials and Candidate Image», Kaid, L. L.; 
Nimmo, D.; Sanders, K. : New Perspectives on Political Advertising, Cardondale Il. : Southern Illinois Press, 210-234.

Devlin, P. L. (1986) : “An Analysis of Presidential Television Commercials, 1952-1984”, Kaid, L. L.; Nimmo, D.; Sanders, K. (1986) : New Perspectives on Political Advertising, Cardondale Il. : Southern Illinois Press, 21-34.

Diamond, E.; Bates, S. (1992) : The Spot. The Rise of Political Advertising on Television, Cambridge : MIT Press.

Diamond, E.; Marin, A. (1989) : «Spots», American Behavioral Scientist, 32, 382-388 .

Ekman, P. (1992) : Telling Lies. Clues to Deceit in the Marketplace, Politics, and Marriage, New York : Norton \& Company.

Garramone, G. (1986) : “Candidate Image Formation”, Kaid, L. L.; Nimmo, D.; Sanders, K. : New Perspectives on Political Advertising, Cardondale Il. : Southern Illinois Press, 235-247.

(1985): "Effects of Negative Political Advertising : the Roles of Sponsor and Rebuttal”, Journal of Broadcasting \& Electronic Media, 29(2), 147-159.

(1984) : "Voter Responses to Negative Political Ads", Journalism Quarterly, 61, 250-259.

Garramone, G.; Smith, S.J. (1984): "Reactions to Political Advertising : Clarifying Sponsor Effects”, Journalism Quarterly, 61, 771-775.

Garramone et al. (1990) : "Effects of Negative Political Advertising on the Political Process”, Journal of Broadcasting and Electronic Media, 34, 299-311.

Gauthier, Gilles (à paraître 1) : "Éthique, argumentation et communication politique. L'éthique de la publicité politique : le cas de la publicité négative" (projet d'article soumis à Éthica).

(à paraître 2): "L'éthique de l'argumentation ad hominem dans les débats politiques télévisés : le cas des débats canadiens et québécois” (projet d'article soumis à Communication).

(1996): "L'éthique de la communication politique : un bilan de la recherche”, Les Études de communication publique, cahier numéro 10, Québec : Département d'information et de communication, Université Laval, 32 pp. [1994]: "Referential Argumentation and Its Ethical Considerations in Televised Political Advertising : The Case of the 1993 Canadian Federal Election Campaign", Argumentation and Advocacy. The Journal of the American Forensic Association, 31(2), 96-110.

Greenawalt, Kent (1995): Private Consciences and Public Reasons, New York: Oxford University Press.

Gronbeck, B.E. (1994): “Negative Political Ads and American Self Images”, 
Miller, A.H.; Grunbeck, B.E. : Presidential Campaigns and American Self Images, Boulder : Westview Press, 60-81.

_ (1992) : “Negative Narratives in 1988 Presidential Campaign Ads”, Quarterly Journal of Speech, 78, 333-346.

Hill, R. P. (1989) : "An Explorer of Voter Responses to Political Advertisements", Journal of Advertising, 18(4),

James, K.; Hensel, P. (1991) : "Negative Advertising : the Malicious Strain of Comparative Advertising", Journal of Advertising, 20, 52-69.

Jamieson, K. H. (1992) : Dirty Politics: Deception, Distraction, and Democracy, New York : Oxford University Press.

Johnson-Cartee, K. S.; Copeland, G. A. (1991) : Negative Political Advertising. Coming of Age, Hillsdale, NJ: Lawrence Erlbaum.

Joslyn, R. A. (1989): "The Content of Political Spot Ads", Journalism Quarterly, 57, 92-98.

(1986) : "Political Advertising and the Meaning of Elections", Kaid, L. L.; Nimmo, D.; Sanders, K. : New Perspectives on Political Advertising, Cardondale Il. : Southern Illinois Press, 139-183.

Kaid, L. L. (1991) : “Ethical Dimensions of Political Advertising”, Denton, R. E. (ed.) (1991): Ethical Dimensions of Political Communication, New York : Praeger, 145-169.

Kaid, L.L.; Johnston A. (1991) : “Negative Versus Positive Television Advertising in U.S. Presidential Campaigns, 1960-1988”, Journal of Communication, 41(3), 53-64. Kern, M. (1989): 30-Second Politics, New York : Praeger.

Louden, A. (1994) : «Voter Rationality and Media Excess : Image in the 1992 Presidential Campaign", Denton, R. E. Jr. : Presidential Campaign : A Communication Perspective, westport, CT: Praeger, 169-187.

Martinez, M.; Delegal, T. (1990) : "The Irrelevance of Negative Campaigns to Political Trust : Experimental and Survey Results", Political Communication And Persuasion, 7 ,

Merritt, S. (1984): "Negative Political Advertising : Some Empirical Findings", Journal of Advertising, 13, 27-38.

Patterson, T.E.; McClure, R.D. (1976): The Unseeing Eye, New York : G.P. Putnam. Pfau, M.; Burgoon, M. (1989): "The Efficacity of Issues and Character Attack Message Strategies in Political Campaign Communication”, Communication Reports, 2, 53-61.

Pfau, M.; Parrott, T.; Lindguist, B. (1992) : "An Expectancy Theory Explanation of the Effectiveness of Political Attack Television Spots : A Case Study", Journal of Applied Communication Research, 20, 235-253. 
Roberts, M.; McCombs, M. (1994) : «Agenda Setting and Political Advertising: Origins of the News Agenda», Political Communication,

Roddy, B.; Garramone, G. (1988) : “Appeals and Strategies of Negative Political Advertising”, Journal of Broadcasting and Electronic Media, 32, 415-427.

Romanow, W.; Soderlund, W.; Price, R. (1991): “Negative Political Advertising: An Analysis of Research Findings in Light of Canadian Practice”, Hiebert, J., Political Ethics : A Canadian Perspective, Toronto : Dundurn Press, 165-193.

Schreier, M.; Groeben, N.; Christmann, U. (1995) : “That's Not Fair!' Argumentational Integrity as an Ethics of Argumentative Communication", Argumentation, 9, 267-289.

Shyles, L. (1986): «The Televised Political Spot Advertisement», Kaid, L. L.; Nimmo, D.; Sanders, K. (1986) : New Perspectives on Political Advertising, Cardondale Il. : Southern Illinois Press, 107-138.

Solum, Lawrence (1993): “Constructing an Ideal of Public Reason”, San Diego Law Review, 30, 730, 754-762.

Spero, R. (1980): The Duping of the American Voter, New York : Lippincott \& Crowell.

Surlin, S.H.; Gordon, T.F. (1977) : "How Values Affect Attitudes Toward Direct Reference Political Advertising”, Journalism Quarterly, 54, 89-98.

Taras, D. (1990) : The Newsmakers : The Media's Influence on Canadian Politics, Scarborough : Nelson Canada.

Tinkham, S. F.; Waever-Lariscy, R. A. (1993) : “A Diagnostic Approach to Assessing the Impact of Negative Political Television Commercials", Journal of Brodcasting \& Electronic Media, 37, 377-399.

Walk, R.; Henley, A. (1970) : The Right to Lie: A Psychological Guide to the Uses of Deceit in Everyday Life, New York : Perter H. Wyden.

Wolton, D. (1989) : "La communication politique : construction d'un modèle”, Hermès, 4, 27-42. 